\title{
A NEW MICROCOMPUTER-BASED APPROACH FOR PILOT DIFFERENTIAL PROTECTION OF TRANSMISSION LINES
}

\author{
Mustahsan Mir' and Patrick J. McCleer ${ }^{2}$ \\ ${ }^{1}$ Umm Al-Qura University, Saudi Arabia and ${ }^{2}$ University of Michigan, Ann Arbor, MI, U.S.A.
}

(Received for publication 18 June 1986)

\begin{abstract}
This paper presents a theoretical and experimental study of a new technique for pilot differential protection of transmission lines. The difference currents obtained from post- and pre-fault line currents, adjusted by calculated charging currents, are used to minimize the desensitizing effect of load and line charging currents. The experimental results are from a laboratory 16-bit microcomputer-based relay that was developed to test some of the features of this new approach. Off-line test results for various types of faults on a lumped parameter long transmission line model and real-time test results for a short transmission line model are presented.
\end{abstract}

\section{INTRODUCTION}

The need for sensitive and selective protection schemes grows in importance as modern power systems increase in size and complexity. A protection scheme should provide a high margin of discrimination between external and internal fault conditions. Pilot differential relaying can provide such margins for the protection of transmission lines. Some of the advantages of pilot differential relaying are [1]:

- sharply defined tripping zone covering the entire line length

- accommodation of high fault resistances

-immunity to power swings and emergency loads.

On the other hand, the general limitations of pilot differential relaying are [1]:

- the desensitizing effect of the capacitive charging currents

- the associated minimum required fault infeed levels

- the need for and degradation of the pilot signal.

Some new types of pilot differential relays have been reported in the past few years. A current-differential pilot relay, based on the principle of percentage differential protection, was developed by Kitagawa et al. [2]. Standard percentage differential relaying, however, is predicated on the condition that the terminal currents at the two ends of a line are in-phase. But, for a transmission line the phase-comparison angles at the two ends are typically in the range of $30^{\circ}-50^{\circ}[1]$, with a corresponding effect on the minimum permissible fault setting. Akimoto et al. [3] have reported a current differential carrier relay (CDCR) which uses a circuit for compensating the erroneous differential current produced by the line charging currents. The operation of this relay, however, is also based on the principle of differential relaying. A "sliced-level" phase-comparison relay has been developed by Okamura et al. [4]. The sensitivity of this relay is affected by both load and line charging currents. For instance, for a solid line-to-line internal fault, the " $L$ sliced-level" must be set larger than one-half the load current, while the "H sliced level" must exceed the sum of "L sliced level" and the charging current in the protected line.

The pilot differential relaying approach presented in this paper compensates the effect of phase-shifts due to line charging currents. Also, the relaying is based on the difference of post- and pre-fault currents to minimize the effect of loads on relay sensitivity. The theory of relay operation and its laboratory test results are presented. 


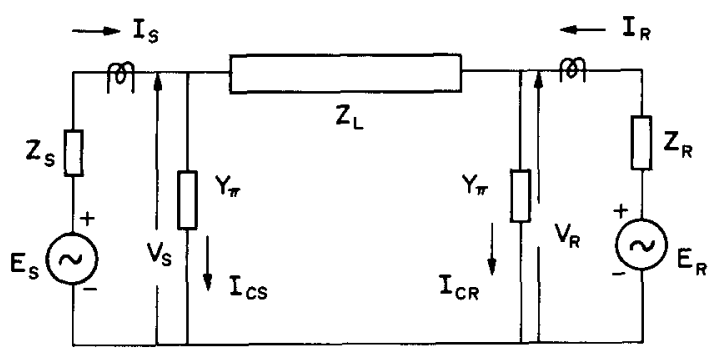

(a)

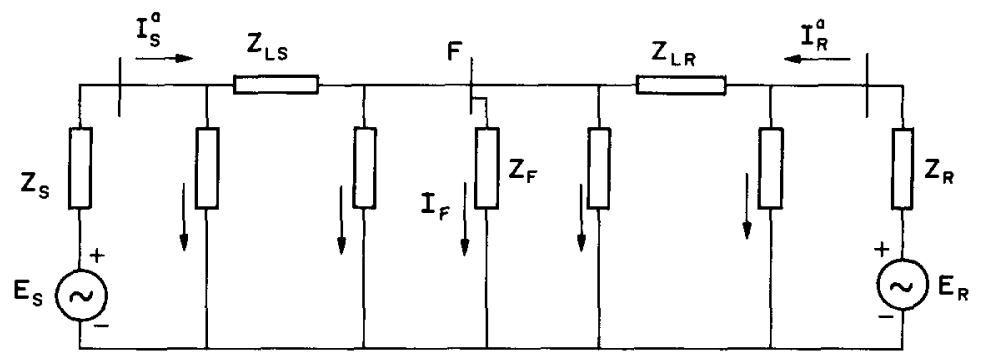

(b)

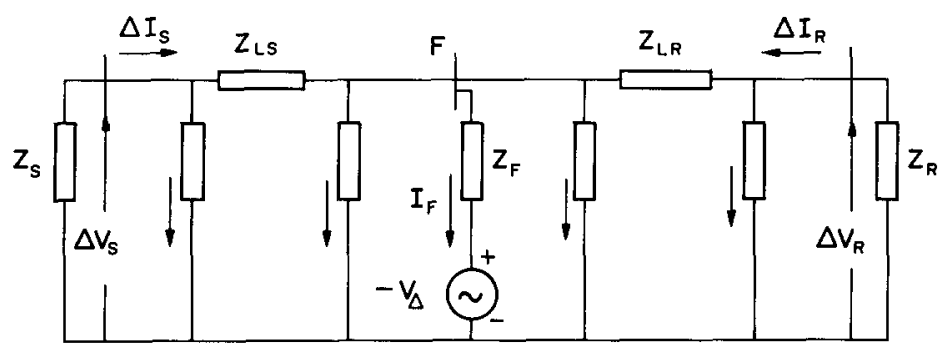

(c)

Fig. 1. Superposition of prefault and delta currents.

THEORY OF RELAY OPERATION

A long transmission line can be represented, in so far as the line voltages and currents at the terminals are concerned, by an equivalent- $\pi$ model as shown in Fig. 1(a):

where

$$
\begin{aligned}
Z_{L} & =Z_{c} \sinh \gamma l \\
Y_{\pi} & =\left(1 / Z_{c}\right) \tanh \gamma l / 2
\end{aligned}
$$

and

$$
\begin{aligned}
\gamma & =\text { Propagation constant of the line } \\
Z_{c} & =\text { Surge impedance of the line } \\
l & =\text { Length of transmission line. }
\end{aligned}
$$

The total charging current, $I_{\mathrm{C}}$, can be written as,

$$
I_{\mathrm{C}}=I_{\mathrm{CS}}+I_{\mathrm{CR}}
$$

where

$$
\begin{aligned}
& I_{\mathrm{CS}}=\left(V_{S} / Z_{c}\right) \tanh \gamma l / 2, \\
& I_{\mathrm{CR}}=\left(V_{R} / Z_{c}\right) \tanh \gamma l / 2
\end{aligned}
$$


The operating principle of the relay can be explained using superposition. For the prefault network considered in Fig. 1(a), the postfault representation, based on the equivalent- $\pi$ model, is shown in Fig. 1(b). The currents flowing in the postfault network can be considered as composed of two components; a prefault component and a fault contributing or $\Delta$-component. The postfault network can therefore be represented by two networks; one that carries the prefault currents as shown in Fig. 1(a), and the other which carries the fault contributing or $\Delta$-currents as shown in Fig. 1(c). The network of Fig. 1(c) will be referred to as the "delta network". The fictitious voltage source shown in Fig. 1(c) is equal in magnitude but opposite in phase to the prefault voltage at the fault point. The protection of a transmission line, based on $\Delta$-currents, is equivalent to protecting a corresponding "delta network".

During normal operation of the transmission line or when an external fault is present,

$$
I_{\mathrm{S}}-I_{\mathrm{CS}}=-\left(I_{\mathrm{R}}-I_{\mathrm{CR}}\right) \text {. }
$$

Forming $\Delta$-currents, that is, the difference currents obtained by subtracting prefault values from suspected postfault values, eqn (6) can be written for external faults as:

$$
\Delta I_{\mathrm{S}}^{\uparrow}+\Delta I_{\mathrm{R}}^{\uparrow}=0
$$

where

$$
\begin{aligned}
& \Delta I_{\mathrm{S}}^{\mathbb{q}}=\Delta I_{\mathrm{S}}-\Delta I_{\mathrm{CS}}=\left(I_{\mathrm{S}}^{\mathrm{a}}-I_{\mathrm{S}}^{\mathrm{b}}\right)-\left(I_{\mathrm{CS}}^{\mathrm{a}}-I_{\mathrm{CS}}^{\mathrm{b}}\right) \\
& \Delta I_{\mathrm{R}}^{\mathrm{\Phi}}=\Delta I_{\mathrm{R}}-\Delta I_{\mathrm{CR}}=\left(I_{\mathrm{R}}^{\mathrm{a}}-I_{\mathrm{R}}^{\mathrm{b}}\right)-\left(I_{\mathrm{CR}}^{\mathrm{a}}-I_{\mathrm{CR}}^{\mathrm{b}}\right) .
\end{aligned}
$$

The superscripts " $a$ " and " $b$ " indicate the currents flowing after and before the occurrence of a fault, respectively. Also, for normal operation,

$$
\Delta I_{\mathrm{S}}=\Delta I_{\mathrm{R}}=0
$$

and

$$
\Delta I_{\mathrm{CS}}=\Delta I_{\mathrm{CR}}=0
$$

and thus for normal operation,

$$
\Delta I_{\mathrm{S}}^{\mathbb{N}}=\Delta I_{\mathrm{R}}^{\mathbb{\mathbb { N }}}=0 .
$$

A differential relay based on delta currents would therefore theoretically require no threshold or restraint to mask the line charging currents.

There would be no discrepancy between the actual value of the charging current flowing through the line and that which is computed using the equivalent- $\pi$ model so long as the system is operating normally or there is an external fault present. For an internal fault, however, the computed value of the charging current is not the same as the actual charging current, which is proportional to the voltage profile along the line. For normal operation or for an external fault, there is only one voltage profile along the entire length of line, but for an internal fault it will have two slopes. The detailed analysis for an internal fault is presented in Appendix 1. It is shown that for an internal fault:

$$
\Delta I_{\text {scal }}^{\mathbb{T}}+\Delta I_{\mathrm{Rcal}}^{\mathbb{\pi}}=I_{\mathrm{F}}^{\mathbb{I}}
$$

where

$\Delta I_{\text {scal }}^{\mathbb{q}}=$ Calculated value of sending-end delta current, adjusted for charging currents,

$\Delta I_{\text {Rcal }}^{\uparrow}=$ Calculated value of receiving-end delta current, adjusted for charging currents,

$I_{\mathrm{F}}^{\mathbb{A}}=$ Apparent fault current.

\section{RELAY TRIPPING CRITERIA}

The relay tripping criterion is based on a comparison of the two delta current phasors, $\Delta I_{\mathrm{S}}^{\mathbb{I}}$ and $\Delta I_{\mathrm{R}}^{\mathbb{T}}$ in accordance with eqns (7), (12) and (13). During normal system operation both delta current phasors are zero; their sum is zero during an external fault and has a 


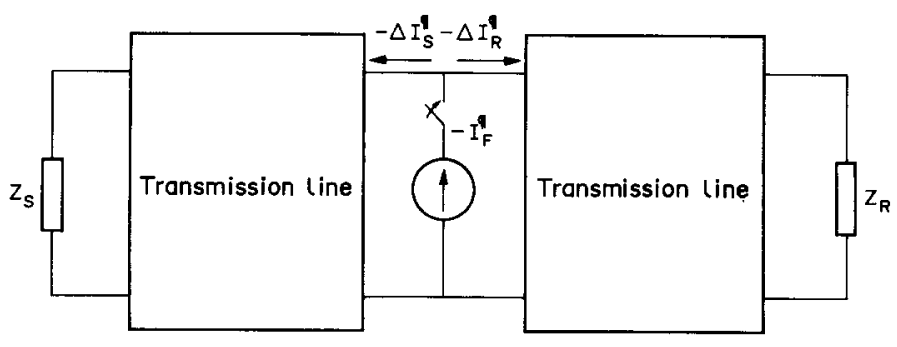

Fig. 2. Current distribution for an internal fault.

value equal to the apparent fault current for an internal fault. In practice, the delta current phasors for normal operation or for an external fault will have small non-zero values due to factors such as A/D conversion, rounding and truncation etc. Therefore, a threshold is introduced in the relay operating characteristics. The value of this threshold is established primarily on the basis of computational inaccuracies. Also, the effect of mutual coupling is considered to avoid tripping of a healthy phase on an internal single line-to-ground fault.

The operation of the relay based on the calculated values of delta current phasors requires transmission of complete information about these delta current phasors. To minimize the required amount of information transmitted between line terminals, another tripping criterion, which requires transmission of angle information only, is described as follows:

Consider the case for an internal fault. As the concept of delta currents is based on the principle of superposition, the apparent fault current $I_{\mathrm{F}}^{\uparrow}$ in eqn (13) can be interpreted as a fictitious current source resulting from an internal fault; and $-\Delta I_{\mathrm{S}}^{\sharp}$ and $-\Delta I_{\mathrm{R}}^{\|}$are two delta currents flowing out of this fictitious source, as shown in Fig. 2. The equivalent impedance on each side of the fictitious current source includes the transmission line as well as the source impedance and is therefore mostly inductive. As a result, the two current phasors $\Delta I_{\mathrm{S}}^{\mathbb{\Phi}}$ and $\Delta I_{\mathrm{R}}^{\mathbb{\uparrow}}$ cannot be more than $90^{\circ}$ out of phase. In practice, the two current phasore, for an internal fault, will have a phase difference much smaller than $90^{\circ}$, as the equivalent impedance angles on both sides of the fictitious current source will be almost similar. For an external fault, however, the two delta current phasors are $180^{\circ}$ out of phase, as indicated by eqn (7). There exists a large buffer zone, unaffected by prefault load and line charging currents, when angle information of the two delta current phasors is used to discriminate between internal and external faults.

\section{ERROR ANALYSIS}

The error in computed fault current due to miscalculation of actual charging currents was investigated using computer simulation of a $500 \mathrm{kV}$ transmission line. The error increases as the fault location moves away from either end of the line, having a maximum value at the middle of the line. The simulation results have confirmed that the error is indeed small. For example, a single line-to-ground fault with a fault resistance of 3.0 p.u. applied in the middle of 200 mile long $500 \mathrm{kV}$ transmission line resulted in the apparent fault current error of about $2 \%$ of the actual fault current. Moreover, it was observed that the magnitude of apparent fault current is larger compared to the actual fault current. The presence of error term in apparent fault current therefore does not practically affect the performance of this relay.

\section{LABORATORY SETUP}

A microcomputer-based relay has been developed for off-line as well as real-time protection of a transmission line model. A 16-bit single-board microcomputer with $4 \mathrm{~K}$ of RAM was used. It has a 8086 microprocessor having a $200 \mathrm{~ns}$ cycle time. The experimental configuration is shown in Fig. 3. The parameters of a $300 \mathrm{kV}$ transmission line and the 


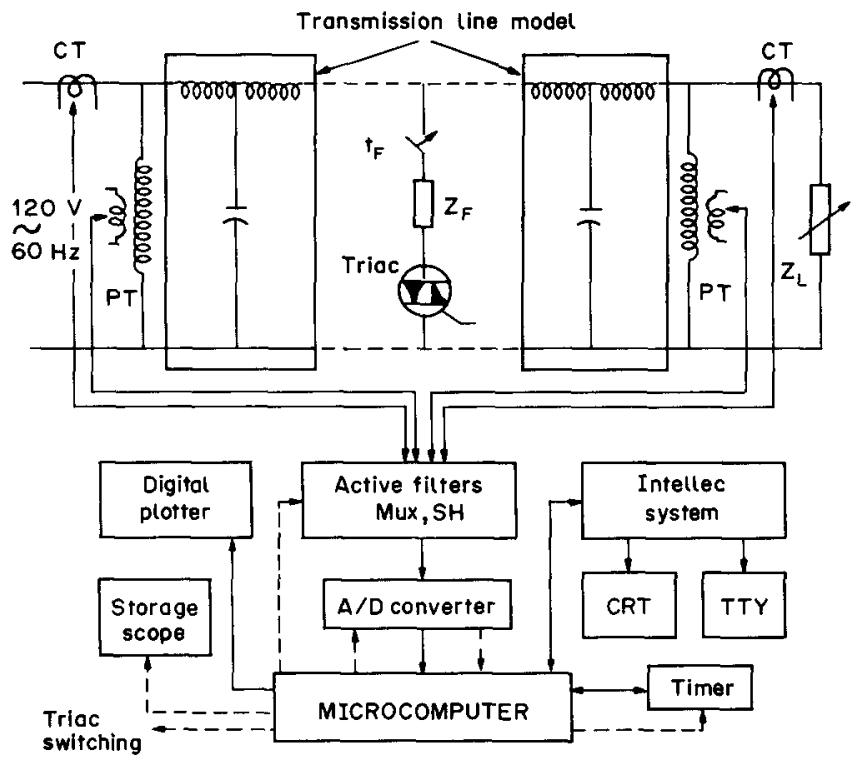

Fig. 3. Experimental configuration. —- Data, --- Control.

corresponding $230 \mathrm{~V}$ model, used in the laboratory tests, are given in Appendix 2. The model is made from discrete components using inductors and capacitors only. The input stage for the microcomputer consists of a $300 \mathrm{~Hz}$, two pole Butterworth low-pass filter, a multiplexer, a sample and hold circuit, and a 12-bit A/D converter. A set of three triacs is used for applying different types of faults on the transmission line through the microcomputer. Faults can also be applied manually by closing a switch. A digital plotter is connected to the microcomputer to plot the delta current phasors. Trigger signals to a storage oscilloscope are also applied through the microcomputer. Prefault, fault and postfault waveforms, along with an indication of the time taken to clear a fault, are displayed and recorded.

\section{RELAYING ALGORITHM}

The current and voltage signals from the two ends of the line are filtered in the input stage, sampled at a rate of 12 samples/cycle, and converted to digital form. The inphase and quadrature parts of the fundamental components are computed using the crosscorrelation technique [5]. Assuming the input signal $X(t)$ to be periodic, the inphase and quadrature parts, $X_{d}$ and $X_{q}$, respectively, of the fundamental component can be computed digitally by cross-correlating it with standard reference sinusoidal signals as:

$$
\begin{aligned}
& X_{d}=\sqrt{ } 2 / N^{*}\left(\sum_{k=1}^{k=N} X_{k-1} \sin 2 \pi(K-1) / N\right), \\
& X_{q}=\sqrt{ } 2 / N^{*}\left(\sum_{k=1}^{k=N} X_{k-1} \cos 2 \pi(K-1) / N\right),
\end{aligned}
$$

where $N$ is the number of samples per cycle and $X_{k}$ is the $K$ th sample of $X(t)$ within a fundamental cycle.

The choice of data-window is a trade-off between relay speed and the effective accuracy of the computed quantities used for trip/no-trip decision making. In general, data-windows of one-fourth of a cycle to one cycle of the fundamental frequency have been considered for computer relaying of transmission lines. Using a data-window of half a cycle, the inphase and the quadrature parts of the fundamental components of the current and voltage signals are regularly computed at each end of the transmission line after every six samples. Using the computed values of the voltages, the charging currents are determined. 
The resultant values of currents at the two ends are determined by subtracting the calculated charging currents from the computed line currents. The prefault values (i.e. values obtained for previous data-window) of the inphase and the quadrature components are subtracted from the corresponding postfault values (i.e. the most recently computed values) to determine the inphase and the quadrature components of the delta currents. A comparison of these delta currents will indicate the presence of a fault in accordance with either of the above mentioned tripping criteria. A control signal is initiated by the microcomputer to clear the fault by switching-off the triacs.

The threshold levels in the operating characteristics of the relay are introduced to take into account the computational inaccuracy of the microcomputer which uses integer arithmetic. This threshold corresponds to $0.75 \%$ of the full scale range of $\mathrm{A} / \mathrm{D}$ converter. For the following experimental studies, the full scale range of the $A / D$ converter corresponds to twice the rated secondary current of the current transformers.

\section{OFF-LINE TEST RESULTS}

The voltage and current signals from a 200 mile long transmission line model are filtered, sampled, converted to digital form and stored in the memory of the microcomputer-based relay. The data stored in the memory is then transferred to a floppy disk. This procedure was repeated a number of times for various types of faults with different fault resistances. Using this data the inphase and the quadrature parts of the resultant delta currents were determined, as explained in the previous section. The relay detected all internal faults with a fault resistance of up to 5.6 p.u. within half a cycle. Some of the resultant delta current phasors, plotted on the $\Delta I_{\mathrm{d}}, \Delta I_{\mathrm{q}}^{\mathbb{I}}$ complex plane, are shown in Fig. 4 for various types of faults. It is observed that for the external faults the resultant delta currents are about $180^{\circ}$ out of phase, whereas their phase difference is much less than $90^{\circ}$ for the internal faults. The presence of a large buffer zone was confirmed by these experiments. Also, during normal operating conditions, the delta currents remained well within the threshold limits.

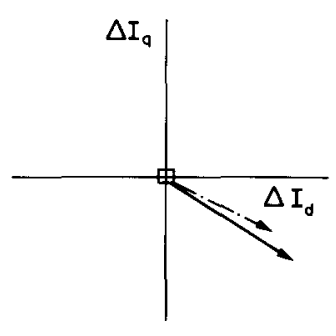

SLG internal fault

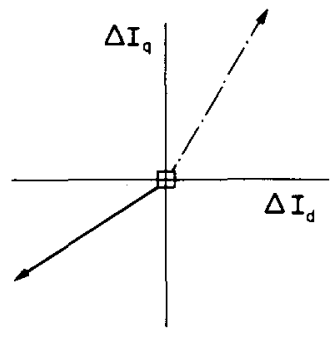

$R_{F}=3.0$ p.u.

SLG external fault

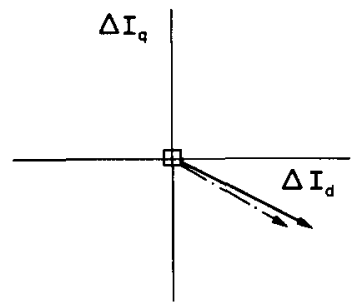

$R_{F}=2.0$ p.u.

LLG internal fault

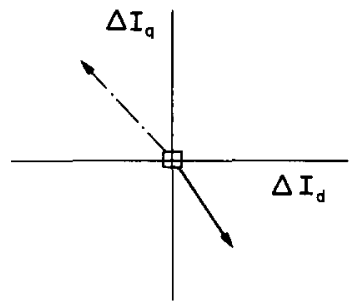

LLG externat foult

Fig. 4. Off-line test results for SLG and LLG faults. 
(a)

Relay operating characteristics

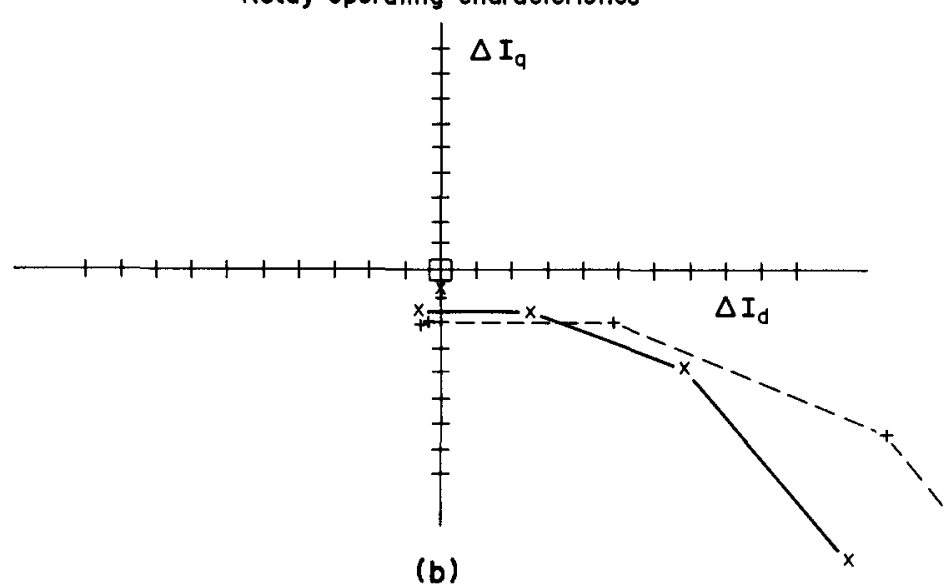

(b)

Relay operating characteristics

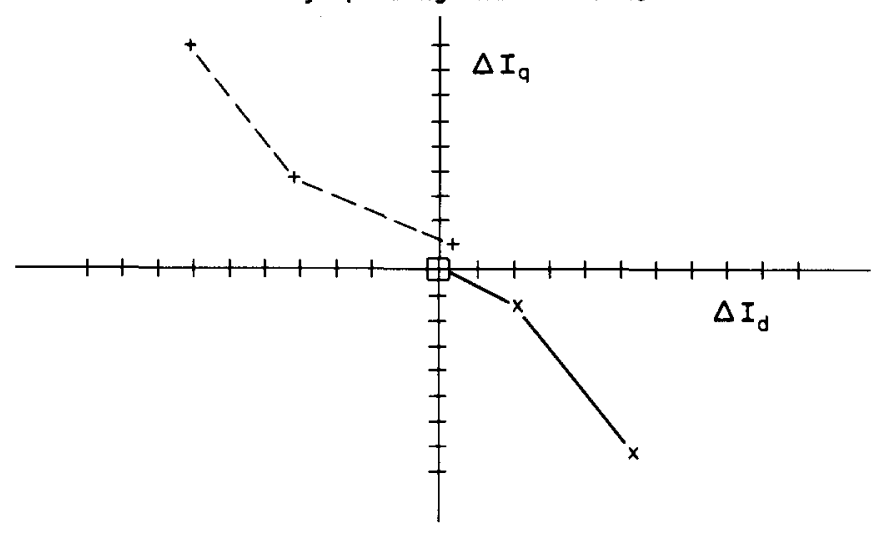

Fig. 5. Real-time test results. (a) SLG internal fault. (b) SLG external fault.

\section{REAL-TIME TEST RESULTS}

The real-time test results were performed for short lines where the effect of charging currents may be neglected. For these lines, the protection can be based upon the delta currents as computed from measured line currents. The protection criteria, however, remain the same as for long transmission lines. This simplification was adopted to test the relaying tripping criteria in real-time, since computation of charging currents as well as line currents could not be performed in real-time by one microcomputer for both ends of the line.

Table 1. Test results for short transmission line

\begin{tabular}{|c|c|c|c|}
\hline Classification & Fault type & $R_{F} /$ phase & Operation \\
\hline \multicolumn{4}{|l|}{ Internal fault } \\
\hline Middle of line & $\begin{array}{c}\text { SLG } \\
\text { LLG } \\
\text { LL } \\
3-\Phi\end{array}$ & $\begin{array}{l}4.0 \text { p.u. } \\
3.5 \text { p.u. } \\
2.0 \text { p.u. } \\
3.0 \text { p.u. }\end{array}$ & $\begin{array}{l}\text { Tripped }(0.5 \text { cycles }) \\
\text { Tripped }(0.5 \text { cycles }) \\
\text { Tripped }(0.5 \text { cycles }) \\
\text { Tripped }(0.5 \text { cycles })\end{array}$ \\
\hline End of line & $\begin{array}{l}\text { SLG } \\
\text { LLG } \\
\text { LL } \\
3-\Phi\end{array}$ & $\begin{array}{l}3.0 \text { p.u. } \\
2.0 \text { p.u. } \\
2.0 \text { p.u. } \\
2.0 \text { p.u. }\end{array}$ & $\begin{array}{l}\text { Tripped ( } 0.5 \text { cycles) } \\
\text { Tripped }(0.5 \text { cycles }) \\
\text { Tripped }(0.5 \text { cycles }) \\
\text { Tripped }(0.5 \text { cycles })\end{array}$ \\
\hline \multicolumn{4}{|l|}{ External fault } \\
\hline & $\begin{array}{l}\text { SLG } \\
\text { LLG } \\
\text { LL } \\
3-\Phi\end{array}$ & $\begin{array}{l}3.0 \text { p.u. } \\
2.0 \text { p.u. } \\
2.0 \text { p.u. } \\
2.0 \text { p.u. }\end{array}$ & $\begin{array}{l}\text { Blocked } \\
\text { Blocked } \\
\text { Blocked } \\
\text { Blocked }\end{array}$ \\
\hline
\end{tabular}




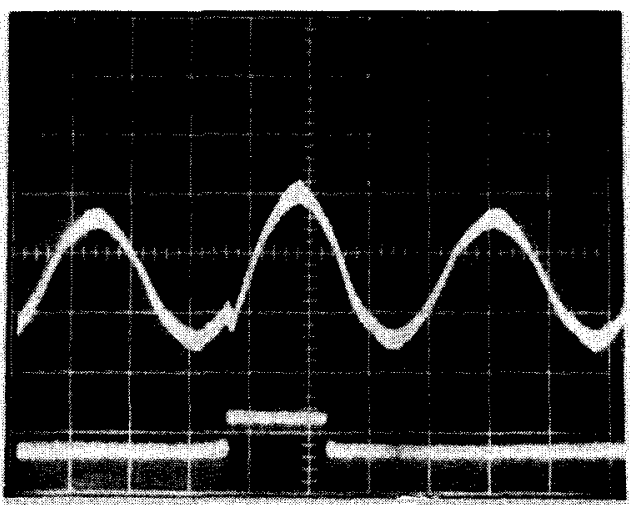

(a) SLG foult

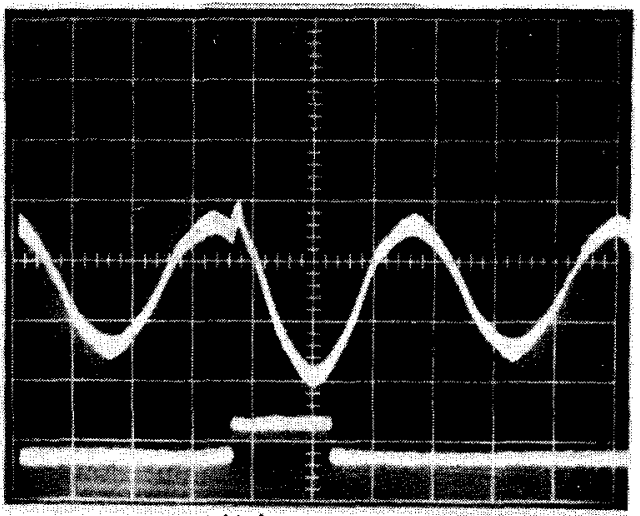

(b) LLG fault

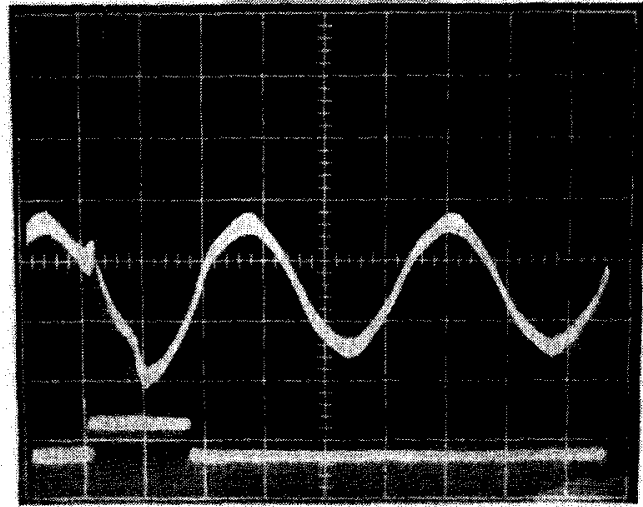

(c) 3-phase fault

Fig. 6. Prefault, fault and post fault current waveforms. (a) SLG fault. (b) LLG fault. (c) 3-Phase-fault.

The delta currents obtained for the short transmission line are plotted in Fig. 5, which shows calculated results at each sampling point. For an external fault, the delta currents are in the opposite quadrants; while they are in the same quadrant for an internal fault. Although a data-window of six samples is used for a trip/no-trip decision, it can be seen from this figure that the internal fault could have been detected at third post-fault sample if a smaller data-window was used or the tripping decision was based on individual sampling points. The waveforms of the current signals are shown in Fig. 6. The rising edge of the signal below these waveforms indicates the initiation of a fault, while the falling edge indicates that the fault has been detected and the signal has been issued to switch-off the triacs. Table 1 summarizes some of the experimental cases studied. The fault type, its location on the transmission line, the fault resistance and the tripping time are indicated. 


\section{CONCLUSIONS}

A new approach for a sensitive and selective protection of transmission lines is presented. The desensitizing effects of load and line charging currents have been minimized. The angle information of resultant delta current phasors is used to determine if the fault is inside or outside the protected zone. Laboratory testing of the proposed scheme has been described. Off-line test results for a long transmission line model and real-time test results for a short transmission line model are presented. The laboratory test results have confirmed the presence of a large buffer zone in the operating characteristics of the relay. This zone, unaffected by load and line charging currents, provides an improved discrimination between internal and external faults. This relaying approach can also be very useful for undergound cables where significant amount of charging currents are present.

\section{APPENDIX 1}

For the analysis of an internal fault, consider that a fault occurs at a distance $l_{1}$ from the sending-end. Let the actual value of the fault current be $I_{\text {Fact }}$. Then:

$$
\left(I_{\mathrm{S}}-I_{\text {CSact }}\right)+\left(I_{\mathrm{R}}-I_{\text {CRact }}\right)=I_{\text {Fact }} \text {. }
$$

Define the errors in the calculated charging currents, $I C_{\text {cal }}$ and actual charging currents, $I C_{\text {act }}$, as:

$$
\xi I_{\mathrm{CS}}=I_{\mathrm{CSCal}}-I_{\mathrm{CSact}} \text {, }
$$

and

$$
\xi I_{\mathrm{CR}}=I_{\mathrm{CRcal}}-I_{\mathrm{CRact}}
$$

Equation (A.1) can then be rewritten as:

$$
\Delta I_{\mathrm{Scal}}^{\mathbb{I}}+\Delta I_{\mathrm{Rcal}}^{\mathbb{I}}=I_{\mathrm{F}}^{\mathbb{I}}
$$

where

$$
\begin{aligned}
\Delta I_{\text {Scal }}^{\natural} & =\left(I_{\mathrm{S}}^{\mathrm{a}}-I_{\text {CScal }}^{\mathrm{a}}\right)-\left(I_{\mathrm{S}}^{\mathrm{b}}-I_{\text {CScal }}^{\mathrm{b}}\right) \\
\Delta I_{\text {Rcal }}^{\mathbb{1}} & =\left(I_{\mathrm{R}}^{\mathrm{a}}-I_{\text {CRcal }}^{\mathrm{a}}\right)-\left(I_{\mathrm{R}}^{\mathrm{b}}-I_{\text {CRcal }}^{\mathrm{b}}\right) \\
I_{\mathrm{F}} & =I_{\text {Fact }}-\left(\xi I_{\mathrm{CS}}+\xi I_{\mathrm{CR}}\right) .
\end{aligned}
$$

Note that $\xi I_{\mathrm{CS}}$ and $\xi I_{\mathrm{CR}}=0$ for no fault, or for an external fault. Also, $I_{\text {Fact }}=0$ for no internal fault present.

\section{APPENDIX 2}

The parameters of the $300 \mathrm{kV}$ line and its corresponding $230 \mathrm{~V}, 10 \mathrm{kVA} 50$ miles long T-model used in the laboratory are as follows:

$300 \mathrm{kV}$ transmission line

$$
\begin{aligned}
& R=0.1 \Omega / \mathrm{mile} \\
& L=2.1 \mathrm{mH} / \mathrm{mile} \\
& C=0.013 \mu \mathrm{F} / \mathrm{mile}
\end{aligned}
$$

$230 \mathrm{~V}$ T-model:

$$
\begin{aligned}
& R=0.035 \Omega \\
& L=0.478 \mathrm{mH} \\
& C=45 \mu \mathrm{F} .
\end{aligned}
$$

\section{REFERENCES}

1. A. R. Van C. Warrington, Protective Relays-Their Theory and Practice. Chapman \& Hall, London (1977).

2. M. Kitagawa, F. Andow, M. Yamaura and Y. Okita, Newly developed FM current-differential carrier relaying system and its field experience. IEEE Trans. PAS 97, 2272-2281 (1978).

3. Y. Akimoto, T. Matsuda, K. Matsuzawa, M. Yamaura, R. Kondow and T. Matsushima, Microprocessor based digital relays application in TEPCO. IEEE Trans. PAS 100, 2390-2397 (1981)

4. M. Okamura, F. Andow and S. Suzuki, Improved phase-comparison relaying with higher performance. IEEE Trans. PAS 99, 522-527 (1980).

5. G. S. Hope, P. K. Dash and O. P. Malik, Digital differential protection of a generating unit-scheme and real-time test results. IEEE Trans. PAS 96, 502-509 (1977). 